\title{
Suplicantes de Esquilo. Ensamble espacio-coral en cuatro movimientos
}

\author{
MARÍA DEL PILAR FERNÁNDEZ DEAGUSTINI \\ UNLP- CONICET \\ mpilarfd@hotmail.com
}

Nuestro objetivo es exponer un esquema compositivo de Suplicantes que logre aprehender su particular técnica estructural, frente a la pauta fija aristotélica. El enfoque pondera el trabajo de TAPLIN (1977) sobre la puesta en escena trágica esquilea, que revolucionó los estudios performativos al demostrar el uso dramático de los movimientos de entrada y salida de los personajes. Dada la singularidad de Suplicantes en cuanto al protagonismo coral y el predominio lírico, sostenemos que el análisis de su estructura impone reformular la propuesta de TAPLIN y considerar las odas corales como parte sustancial de los actos (WILES, 1997).

Estructura / Poética / Taplin / Suplicantes / Esquilo

\section{AESCHYLUS' SUIPPLIANTS. SPACIAL-CHORAL ENSAMBLE IN FOUR MOVEMENTS}

Our aim is to expose a Supplices' compositional scheme that manages to apprehend its characteristic structural technique, facing the fixed Aristotelian pattern. The approach analyses the work of TAPLIN (1977) relating to Aeschylean tragic performance, that radically changed the performative studies by demonstrating the dramatic use of character's exits and entrances. Given the uniqueness of Aeschylus' Supplices regarding the leading role of the chorus and the predominance of lyrics, we hold that the analysis of its structure imposes us to revise TAPLIN's proposal and to consider that the choral odes are a substantial part of the acts (WILES, 1997).

Structure / Poetics / Taplin / Supplices / Aeschylus 
I hope to show that the whole subject of the formal structure of Greek tragedy is worth reviving from its state of moribund stagnation; and to do something towards showing how this kind of analysis is a way of approaching the artistic shaping and formation of a tragedy, and hence its critical interpretation (TAPLIN, 1977: 60)

\section{Introducción}

La liricidad y el protagonismo del coro han sido las características que más han influido en la separación de Suplicantes del resto de las tragedias conservadas, lo que le valió, antes del hallazgo del papiro que puso en cuestión su conjeturada datación temprana, la calificación de "primitiva» ${ }^{1}$. Esta arbitrariedad, condicionada por el incomparable planteo compositivo de la obra, restringió la estimación de su calidad dramática. Consecuentemente, el acercamiento a Suplicantes a partir de un patrón dramático estandarizado asociado a la tradición aristotélica ha predispuesto juicios interpretativos adversos a lo largo de los siglos XIX y XX. Por ejemplo, en la señera edición crítica de Cambridge, Thomas TUCKER sostiene que Suplicantes fracasa en el efecto dramático, pues no hay para él ninguna acción apasionante en la obra y, pese a su admirable poesía, habría resultado "chata" sin el efecto espectacular de un coro multitudinario ${ }^{2}$. A pesar de la antigüedad de esta apreciación y de otras tantas posibles citas que redundarían sobre el mismo dictamen ${ }^{3}$, el derrotero de los estudios sobre Suplicantes ha sido guiado por las miradas de prestigiosos eruditos, enceguecidos por las polémicas en torno a la datación de la tragedia.

En medio de semejantes interpretaciones aciagas, el criterio de KITTO ha sido una excepción. En 1939, la primera edición del libro Greek Tragedy comenzaba con la aseveración de que Suplicantes era la primera obra conservada del drama europeo. Dos décadas después, en la tercera edición del libro, el autor rectificaba esta conjetura, reconociendo la existencia y validez del papiro de Oxirrinco 2256.3. Sin embargo, la enmienda no ha tenido injerencia en la alteración del memorable título del capítulo inicial, que involucra el estudio de la obra. Suplicantes continuó siendo, a los ojos de KITTO, una «tragedia lírica». Sin duda, esta renovada edición refleja el principio que, según el autor, anima a toda obra de arte: la íntima conexión entre forma y contenido ${ }^{4}$. El compromiso continuo de KITTO de mostrar a la tragedia griega como un fenómeno cultural vivo y al dramaturgo como productor de un tipo de arte diseñado para ser visto explica no solo por qué el autor decidió conservar un título que describe perfectamente la esencia de Suplicantes, sino también por qué un título semejante no va en desmedro de la excelencia dramática. Afirmaba KITTO en 1961:

By all means let us think some passages in the play clumsy; nevertheless the greater part of it handles a profoundly tragic situation -and a familiar one- with immense power. Our first duty is to discover where Aeschylus laid the emphasis; we may assume that he built the play as he felt it. Certainly, those 
who find it undramatic cannot tell us, except by accident, what it is about, for they will not have seen the drama ${ }^{5}$.

En los albores de los 60, KITTO colocaba en el centro de la polémica sobre la calidad dramática de Suplicantes un elemento despreciado por quienes han sentenciado su inmadurez: la dimensión visual, que, asociada a la puesta en escena, recobra su peso en la construcción de significado. De este modo, este acreditado estudioso reveló que una obra aparentemente llana en la lectura descubre su sofisticación cuando se reconstruye su representación. A pesar de su clarividencia, KITTO no ha profundizado en el análisis de la puesta en escena de Suplicantes. No obstante, años más tarde, su precursora perspectiva sobre el drama griego clásico fue cumplida por la revolucionaria labor de TAPLIN $(1977,1978)$, quien acuñó el concepto de «tragedia en acción».

El presente artículo pretende señalar y revertir el instalado menosprecio de Suplicantes. Evidentemente, gran parte de la crítica se ha concentrado en juzgar la complejidad de la trama de la obra, identificándola con el contenido y perdiendo de vista que la forma a través de la cual se comunica el texto es el espectáculo, que hace del discurso escrito una experiencia audiovisual, una tragedia. Por lo tanto, si bien la trama podría parecer "primitiva" por su simpleza, no se verifica lo mismo si se pondera la complejidad de su composición como obra teatral. En Suplicantes, Esquilo puso en práctica un uso efectivo e integral del aspecto visual, capaz de transmitir los momentos de mayor tensión dramática. Nuestro objetivo es exponer un esquema compositivo del drama que logre aprehender su particular técnica estructural, es decir, su singularidad en cuanto al protagonismo coral y el predominio lírico.

El análisis del diseño compositivo de una obra teatral demanda la detección de las acciones más significativas del drama. En relación con esta materia, continúa siendo inspirador el trabajo de TAPLIN sobre el uso dramático de las entradas y salidas de los personajes, aunque resulte necesario realizar ciertas enmiendas. Si bien la fórmula estructurante que se propone en este artículo recupera el planteo innovador de este estudioso, resulta necesario señalar dos cuestiones. Por un lado, TAPLIN no ha encuadrado sus interpretaciones sobre los ingresos y egresos de los personajes en una construcción formal integradora que permita visualizar y aprehender cierta "lógica" compositiva, de las obras en general y de Suplicantes en particular, y la repercusión que tiene la dimensión visual sobre la comunicación de la totalidad de la trama. Por otro lado, tal como ha señalado certeramente WILES en su exhaustivo trabajo sobre la trascendencia del espacio en la performance y el significado teatral, el coro está ausente en las páginas de TAPLIN ${ }^{6}$. Sobre la base de ambas publicaciones, en definitiva, la presente propuesta consiste en gestar una mirada estructural superadora sobre la tragedia, que logre continuar el camino iniciado por la corriente performativa pero que, simultáneamente, respete su idiosincrasia lírica. Por lo tanto, si bien la atención está concentrada en los desplazamientos 
de los personajes (incluido, entre ellos, el coro), el planteo se incorpora a una concepción del teatro griego clásico enraizada más en la tradición platónica que en la aristotélica ${ }^{7}$.

La lógica del método consiste en pasar de la dimensión temporal a la espacial. En el «teatro de plataforma», que Anne UBERSFELD ha sabido contrastar con el «teatro de proscenio», la organización está basada en las relaciones espaciales visibles para el espectador. El límite no está en la división entre actor y audiencia, sino en la demarcación entre la plataforma y todo lo que está fuera de la vista. UBERSFELD afirma que, en el teatro de proscenio, la organización espacial está unida a la ficción dramática, al espacio imaginado del cual el espacio escénico es meramente una "traducción" concreta, mientras que en el teatro de plataforma el espacio se organiza en relación con los cuerpos de los actores que lo invisten. De este modo, el acento está puesto más en el cuerpo del performer, a lo que UBERSFELD llama ludus, que en el referente, el otro lugar implicado o mímesis ${ }^{8}$, aunque ambos mecanismos estén presentes en todas las obras. En este trabajo, pretendemos examinar algunas de las transformaciones «lúdicas» del espacio que Esquilo parece haber llevado a cabo en Suplicantes.

\section{Esquema compositivo de Suplicantes}

Antes del descubrimiento del papiro de Oxirrinco, Suplicantes era considerada no solo la tragedia más antigua entre todas las conservadas, producida en el 490 a. C., sino también la única tragedia representativa de la primera etapa del género ${ }^{9}$. Esta presunción casi unánime estaba basada primordialmente en la impresión de que la técnica dramática de la obra era arcaica (dado que carece de prólogo y desarrolla un argumento aparentemente simple) y en que las posibilidades ofrecidas por el uso del segundo actor (intérprete de Dánao y el heraldo de los Egipcios) no habían sido adecuadamente desarrolladas todavía, ya que la lírica coral predominaba sobre el diálogo entre actores. Como la predominancia de elementos corales había sido asociada al drama temprano a partir de la teoría desarrollada en Poética sobre el origen coral de la tragedia, para los críticos modernos fue natural percibir esta particularidad de la obra como una característica primitiva. Efectivamente, Suplicantes no se adapta al esquema de alternancia entre secciones episódicas y estásima.

Apartarse del patrón estructural inalterable y artificial aristotélico implica concebir un sistema de análisis que permita aprehender la estructura compositiva particular de cada obra dramática. La propuesta de TAPLIN ha sido absolutamente innovadora en este sentido, al sostener que el marco estructural de una obra dada está basado en (1) la interacción de dos modos principales de presentación dramática (discursos de actores y canciones del coro) junto con (2) la articulación de la acción a través de las entradas y salidas de los personajes. Según el autor, la unidad formal mínima que se repite de manera sucesiva en la representación trágica es ingreso de actores-acto-salida de actores-canción coral estrófica. Tras la canción, esta unidad se reitera en cantidades variadas según cada tragedia ${ }^{10}$. 
TAPLIN ha hecho un importante aporte al incluir, entre los acontecimientos/ recursos fundamentales de la performance, los movimientos de ingreso y salida de los personajes. Sin embargo, lo cuestionable de la propuesta radica en la siguiente aseveración:

Within this frame (which is far simpler than the practice) everything except the strophic choral songs would properly come within the acts - that is to say, all kinds of speech, monody, duet, lyric dialogue, astrophic lyric, etc. all come within the acts ${ }^{11}$.

Es decir que, para TAPLIN, las odas corales se encuentran reducidas a la categoría de canciones divisorias («act dividing song») y, como consecuencia de su trascendencia en términos estructurales, su función dramática en el desarrollo de la trama y del espectáculo queda relegada.

Si bien es indiscutible que la alternancia entre discurso dialogado y canciones estróficas corales constituye un síntoma de algún tipo de modificación en la acción dramática, debe rectificarse el supuesto de que las odas no formen parte de los actos. Esta aproximación al drama mantiene resabios aristotélicos ${ }^{12}$. Como ha sido demostrado en publicaciones anteriores ${ }^{13}$, las odas corales de Suplicantes constituyen hitos fundamentales de la acción dramática, no un mero discurso lírico que complementa la actividad actoral ${ }^{14}$. La prevalencia de versos líricos en Suplicantes constituye una excepción dentro de las obras conservadas que invita especialmente a revisar este tipo de principios establecidos. No obstante, el cambio de paradigma respecto del drama clásico griego incluye la tragedia y la comedia como géneros corales, prescindiendo del volumen de lírica presente en cada una de las obras conservadas.

Numerosos estudios posteriores a las publicaciones de The Stagecraft of Aeschylus y Tragedy in Action han promovido la reevaluación radical de las obras como eventos corales, recolocando al coro en el centro interpretativo de los «textos» dramáticos, entendidos ya, efectivamente, como insumos de una experiencia ${ }^{15}$. Esta mirada ha quebrado abruptamente una extensa tradición académica de raigambre aristotélica, modelada por la idea de que el drama ático alcanzó su perfección cuando se separó de sus orígenes líricos (Poética 1449ª10-15), de que la tragedia es «la representación de una acción», cuyas partes fundamentales son la trama y los personajes, mientras que la poesía lírica y el espectáculo quedan relegados a lo accesorio (Poet. 1450 a-b), que el coro debe ser considerado como uno de los actores $\left(1456^{\mathrm{a} 25}\right)$ y que, a partir de Agatón, las odas corales son consideradas como embólima, interludios, separados de la trama (Poet. 1456a30).

TAPLIN ha revolucionado los estudios performativos demostrando la significación de las salidas y entradas de los personajes, pero ha dejado al coro fuera de su criterio estructural. Precisamente, como señalamos, Suplicantes de Esquilo resulta una tragedia provocativa, porque nos insta a liberarnos de la asunción de que, en términos temporales, las odas corales constituyen "interludios". Una vez que reconocemos el principio de que actores y coro compartían el mismo espacio, la 
orchestra, resulta consecuente que las partes cantadas y las dialogadas de la obra constituían un todo integral:

Any Greek tragedy could be examined to make the same point, that there is no sharp spatio-temporal demarcation of choral ode from action. The purpose of the ode is not to offer philosophical reflection, emotional relief, or the pleasure of verbal pictures. It is meshed with the action, but creates the possibility of seeing the action in terms that are not monocular and rationalistic ${ }^{16}$.

Una estructura formal auténtica, que respete las particularidades de cada composición, no debe prefijar ningún tipo de molde compositivo, sino limitarse a considerar el uso de los recursos técnicos disponibles. En el caso especial de Suplicantes, sostenemos que (1) la alternancia discursiva se produce entre coloquio y soliloquio ${ }^{17}$ y que (2) existe una relación desigual entre el peso dramático de las entradas de los personajes en relación con el de las salidas. Por otro lado, la particular sintaxis espacial de la obra, con el altar como «eje vertical ${ }^{18}$ de la performance, tensionado entre Argos y Egipto como espacios en confrontación, impone otra mirada ${ }^{19}$.

Para TAPLIN, aquellas entradas que inauguran un nuevo acto y aquellas salidas que retiran a los personajes al final de un acto son estructuralmente importantes, porque son los movimientos que llenan y vacían el escenario ${ }^{20}$. Pero, en Suplicantes, el escenario, el espacio de representación que ocupan los actores, coincide con el del coro. Por lo tanto, nunca está vacante. El coro-personaje permanece pendiente, durante toda la obra, de aquellos que vienen a su encuentro. Por este motivo, la estructura formal de esta tragedia debe ser definida a partir de los ingresos de los personajes a escena, que relacionan, por un lado, texto y acción, y, por el otro, acción y «significado teatral», en términos de WILES ${ }^{21}$.

En primer lugar, resulta necesario precisar en qué consiste una «entrada», ya que, si bien significaría el movimiento del personaje que lleva a un actor hacia el campo de visión de la audiencia, el acontecimiento debe ser especialmente considerado dentro del contexto de representación que ofrecía el drama griego clásico. Por un lado, en el teatro clásico, orchéstra y eísodoi implicaban distancias considerables, por lo tanto, es difícil determinar con exactitud el momento en el que un actor cruzaba la línea entre lo visible y lo imperceptible para la audiencia. Por otro lado, dado que la mayoría de las entradas eran indizadas por las palabras de aquellos que estaban en escena (coro o actores), no siempre estos índices resultarían simultáneos con la acción ${ }^{22}$. Sin embargo, en la estructura de Suplicantes que proponemos, articulada alrededor de los movimientos de ingreso, se fija un verso particular para cada acontecimiento ${ }^{23}$. La elección está basada en un criterio dramático, por lo tanto, no implica que la entrada sea concebida como instantánea, ni como el momento en que el actor se hacía visible ante la audiencia. Por este motivo, resulta más apropiado llamar «llegadas» ${ }^{24}$ a estos puntos de inflexión en la acción, expresando que se trata de movimientos que se cumplen en un tiempo prolongado. 
La elección del motivo de súplica es decisiva en la composición tanto de la red de personajes, como de los potenciales conflictos. Si los movimientos de llegada y partida repercuten en la composición formal de una obra, el motivo temático es otro de los factores determinantes de la estructura. En principio, el Altarmotiv impone la trascendencia de dos llegadas previsibles, la del salvador y la del perseguidor ${ }^{25}$. En términos compositivos, ambas acciones son puestas en relieve a través de la disposición espejada de los «anuncios extendidos» (176-233; 710-733) ${ }^{26}$, que funcionan como un método de preparación que crea expectativa y coloca al evento dentro del contexto dramático, asignándole su valor en la puesta en escena ${ }^{27}$. Considerando que los movimientos escénicos en la tragedia griega clásica eran escasos pero importantes, su gestación anticipada contribuye notablemente a la continuidad, economía y dirección de la obra, así como a la organización de una estructura. En Suplicantes, el uso de los anuncios extendidos como recurso de preparación de las dos llegadas capitales promueve una primera apreciación formal: la división de la obra en dos partes. Sin embargo, el peso de estos acontecimientos en la arquitectura de la obra es todavía mayor, por la complejidad que adquieren en el nivel de la trama.

Precisamente porque las técnicas de anticipación y preparación eran tan cuidadosamente controladas por los dramaturgos, su empleo u omisión ofrecía oportunidades especiales de suscitar expectativa o sorpresa ${ }^{28}$. Reconocer que estas cuestiones formaban parte de la discreción del compositor intima al crítico a indagar por qué los movimientos de los personajes fueron arreglados de una forma y no de otra. En Suplicantes, resulta necesario reflexionar sobre cada una de las llegadas de manera aislada, discriminando la trascendencia dramática en cada caso, el momento en que ocurren (tanto en relación con el contexto como con la vaguedad o precisión del evento) y el tratamiento visual del movimiento (dirección, preparación). Asimismo, en relación con la composición de las distintas partes, es necesario tener en cuenta su brevedad o longitud y las relaciones simétricas o divergentes respecto de otras escenas.

\section{Suplicantes consta de seis movimientos de llegada al eje vertical, marcado por el altar ${ }^{29}$ :}

\begin{tabular}{l|l|l}
\hline & Verso & Personajes \\
\hline 1. & 1 & Llegada de Danaides y Dánao \\
\hline 2. & 234 & Primer regreso de Dánao \\
\hline 3. & 600 & Llegada de Pelasgo (junto a los Argivos) \\
\hline 4. & 825 & Llegada del heraldo de los Egipcios \\
\hline 5. & 911 & Regreso de Pelasgo \\
\hline 6. & 980 & Segundo regreso de Dánao (junto a los Argivos) \\
\hline
\end{tabular}

Entre estos acontecimientos, tres resultan irrefutablemente indispensables en el desarrollo de una tragedia compuesta sobre la base del Altarmotiv: en primer lugar, el arribo de los suplicantes al lugar sagrado, que implica el comienzo del ritual; 
luego, las llegadas del potencial protector y del perseguidor ${ }^{30}$. En la tragedia, estas tres llegadas están especialmente destacadas. El arribo de los suplicantes coincide con los anapestos de marcha del coro, que componen la parte dinámica de la oda inicial (vv. 1-39). El hecho de ser una obra carente de prólogo intensifica la recepción del movimiento escénico, como consta en el análisis de esta escena ${ }^{31}$. Las llegadas del protector y del perseguidor, Pelasgo y el heraldo, se distinguen por su preparación a partir de los «anuncios extendidos» (176-233; 710-733), simétricos en cuanto a su léxico, sintaxis y escenificación ${ }^{32}$.

La secuencia de estas tres llegadas determina tres instancias diferentes de la hiketeia: 1. entre la llegada de los suplicantes y la llegada del salvador tiene lugar la súplica con el destinatario divino, conformado por el conjunto de los dioses representados en el altar; 2. entre la llegada del salvador y la del perseguidor se ejecuta la súplica ante el destinatario humano, Pelasgo; 3. tras la llegada del perseguidor, el heraldo, se pone a prueba la efectividad de la institución de la hiketeia.

La novedad de la trama reside en el hecho de que la obra no consiste solamente en las circunstancias en las cuales las suplicantes están en condiciones de abandonar el refugio sagrado, sino en las condiciones en las que efectivamente resuelven hacerlo: tras consultar a su padre, que vuelve a ser convocado a escena por pedido de las jóvenes ${ }^{33}$. Teniendo en cuenta esta variante, cobra importancia otra llegada: el segundo regreso de Dánao en el verso 980. La envergadura dramática del movimiento es señalada a través de distintos recursos. El primer síntoma lo constituye la irregularidad que la llegada ${ }^{34}$ introduce dentro del patrón temático, dado que resulta incongruente con el Altarmotiv. En segundo lugar, debe señalarse el hecho de que dicha incongruencia no implica desconexión absoluta de la trama, pues el regreso es preparado por las Danaides, que solicitan la presencia de su padre como requisito para apartarse del altar y encaminarse hacia Argos (968-971). Por último, el segundo regreso de Dánao se destaca por la compañía de un personaje colectivo secundario, los Argivos, que no solo exaltan al personaje del padre, sino que invitan a homologar esta tercera llegada de Dánao con la llegada inicial de Pelasgo, el potencial salvador. La estructura formal de la obra, entonces, instala un sentido fundamental: se presenta a Dánao como el salvador, acompañado por los representantes de Argos. De este modo, puede verse el primer indicio de una composición de tipo anular: el padre, como consejero y líder de la formación coral (11), es el verdadero protector y benefactor a los ojos de las Danaides, porque supo decidir ( $̇ \epsilon \varepsilon ́ \kappa \varrho \alpha \nu \varepsilon v, 12)$ el camino correcto para lograr la liberación del dominio de los hijos de Egipto. Además de posibilitar el cierre de la coyuntura trágica vinculada con la obtención del asilo y la huida definitiva de la desmesura de los parientes, la llegada de Dánao permite reavivar el conflicto original vinculado con el rechazo del gámos ${ }^{35}$, que se repone en la última escena de la obra y queda pendiente de definición ${ }^{36}$.

La significación de los dos movimientos de llegada restantes, el primer regreso de Dánao (600) y el único regreso de Pelasgo (911), está vinculada con el tratamiento dramático que realiza Esquilo en relación con las insustituibles llegadas del Altarmotiv. 
El primer regreso de Dánao se inserta en un contexto dramático específico, porque la llegada de Pelasgo (234) no constituye propiamente una garantía de salvación, debido a que el refugio de las Danaides debe ser convalidado por el pueblo de Argos. Es destacable el hecho de que, si bien la llegada de Dánao para comunicar a sus hijas la decisión de la asamblea es, técnicamente, un regreso, simultáneamente resulta un movimiento novedoso: primero, porque se trata de su primera llegada desde Argos; segundo, porque su discurso conlleva, de algún modo, la comparecencia escénica de un agente externo, el pueblo. Por lo tanto, esta llegada es también trascendental, en la medida en que representa el arribo de la salvación.

Sin duda, el haber estado en escena exime a Dánao de ser presentado, pero no por ello deja de ser sorpresiva su asistencia. Dánao no solo no es anunciado por las Danaides, sino que, además, su intervención discursiva, que es inmediatamente posterior a la segunda oda coral, no está encabezada por introducción alguna. En la primera línea de su discurso (600), Dánao conmociona a destinatarios internos y externos con la buena nueva del asilo.

El regreso de Pelasgo tiene en común con el primer regreso de Dánao el elemento sorpresa. A pesar de que el rey arriba al altar para responder al pedido de auxilio de las Danaides (835) ${ }^{37}$, su llegada sobreviene a último momento (911), cuando las Danaides y el espectador consideran que ya no hay escapatoria ante los violentos apremios del heraldo. Por otro lado, el regreso de Pelasgo también habría resultado extraño para el espectador, si se acepta el hecho de que, a diferencia de su escena anterior, esta vez habría llegado sin los guardias. La soledad del rey anticipa que su socorro no es más que el inicio de una escena de salvación, hazaña que consuman Dánao y los guardias (980).

\section{El análisis de los seis movimientos de llegada deriva en las siguientes conclusiones:}

1. La obra se organiza alrededor de cuatro «llegadas fundamentales» $(1 ; 234 ; 836$; 980) y dos «llegadas complementarias» $(600 ; 911)$.

2. En relación con el contexto dramático en que se producen, como pretende indicar el concepto utilizado, las «llegadas complementarias» rematan las escenas iniciadas por las llegadas inmediatamente precedentes (234; 825). El juego dramatico es el mismo, aunque las situaciones no resulten idénticas: ante una situación de riesgo/ inseguridad (llegada del potencial amigo; llegada del enemigo), las «llegadas complementarias» definen el bienestar de las protagonistas (noticia del asilo; expulsión del heraldo).

3. Las cuatro «llegadas fundamentales» dividen la obra en cuatro partes: súplica divina- súplica humana- amenaza efectiva- nuevo conflicto. Las primeras tres desarrollan el conflicto por el logro del asilo en la ciudad receptora, para librarse de la condición inicial de fugitivas (5) y recuperar la pertenencia a una comunidad (609 y ss.); la última sienta las bases para una nueva coyuntura trágica, esbozada en la exposición de la causa que originó la determinación del autoexilio (el rechazo del 
gámos con los hijos de Egipto), incluyendo una alternativa inédita a los ojos de las protagonistas: la posibilidad del gámos deseado ${ }^{38}$. Los dos conflictos, súplica por el asilo y gámos, están ligados a dos espacios distintos: la condición de fugitivas y suplicantes obliga a las Danaides a encomendarse al amparo del altar; la condición de metecas exige a las jóvenes el abandono del refugio y la incorporación a la comunidad de Argos, en la que resulta imprescindible aceptar sus responsabilidades femeninas.

4. El análisis de la modalidad de las llegadas aporta dos datos:

La reflexión sobre la dirección permite distinguir entre llegadas desde el mar (Egipto) hasta el altar, en la costa de la ciudad, y llegadas desde Argos hasta el mismo sitio. Como ha observado WILES, en toda tragedia griega los dos eísodoi articulan una oposición entre dos locaciones fuera del escenario, opuestas tanto topográfica como simbólicamente ${ }^{39}$. Las dos llegadas que se producen desde Egipto $(1 ; 825)$ implican una amenaza para la ciudad receptora: los suplicantes colocan a Argos en una coyuntura irresoluble; la actitud de los Egipcios al llegar impone, como única posibilidad de solución del conflicto, la guerra. Por lo tanto, la interpretación de la dirección de los movimientos escénicos impone un sentido dramático: aquello que no es griego es peligroso.

La reflexión sobre la preparación de los movimientos coincide con la jerarquización de las llegadas: las «fundamentales» (excepto la primera, por corresponderse con la primera escena de la obra) son anticipadas (anuncios extendidos; demanda de las Danaides por la presencia de su padre); las «complementarias» no lo son. Esto permite distinguir entre acontecimientos sobre los que se crea expectativa y acontecimientos que sorprenden. Los momentos más significativos aprovechan el suspenso y, a través de este, se instalan en la obra, apropiándose del tiempo dramático ${ }^{40}$; las «llegadas complementarias» llevan la realización de la tragedia a un nivel más complejo, pero se presentan como acontecimientos fugaces que solo toman el tiempo indispensable para cumplir su cometido. La observación detenida de los seis movimientos de llegada resulta en la determinación del siguiente esquema compositivo: 


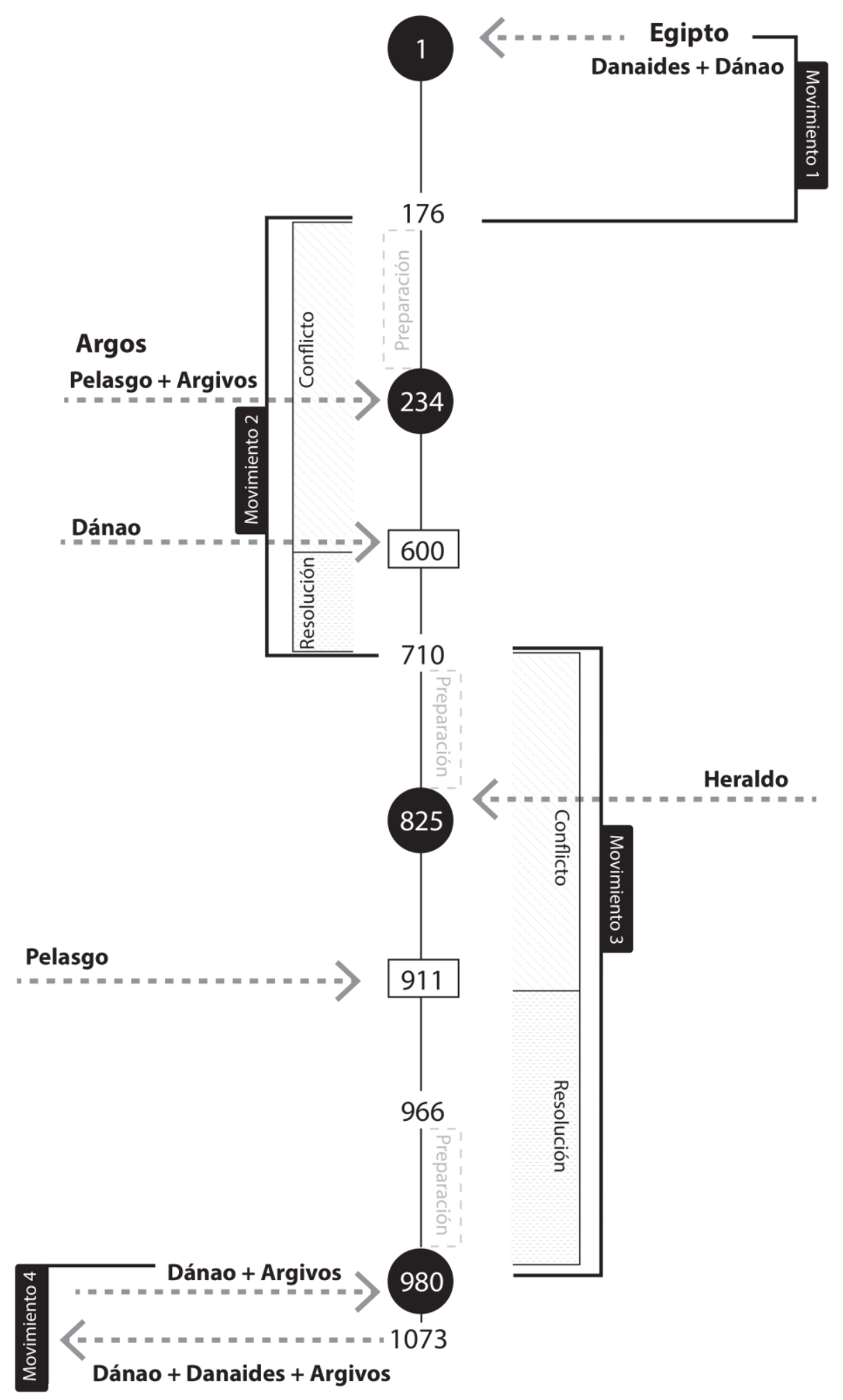

Como muestra el diagrama, Suplicantes presenta una estructura anular, acorde al procedimiento compositivo propio de Esquilo, dividida en cuatro «movimientos». «Movimiento» es el concepto seleccionado para nombrar cada una de las cuatro partes del drama tanto en el sentido estricto de actividad, como en el derivado que se refiere a una parte de una composición musical. El primer sentido supone una acción de desplazamiento de los personajes, coincidente con la propuesta de 
TAPLIN. El segundo atesora un sentido propio del "análisis musical" que, en disidencia con TAPLIN, pretende destacar la impronta de estilo y de sentido (ritual y, por ello, dramático) que las odas corales aportan a la obra. En la música clásica, un movimiento es una parte de una composición más amplia previsto para ser ejecutado en sucesión con otros, aunque propiamente posea un inicio y un final ${ }^{41}$. Por lo tanto, es una parte de una secuencia que conlleva un sello discursivo diferente, vinculado con el canto y la danza corales.

Cada uno de los cuatro movimientos que componen la obra gira alrededor de una llegada fundamental al eje vertical: los suplicantes (Danaides y Dánao), el protector civil o próxenos (Pelasgo), el perseguidor (heraldo de los Egipcios), el protector natural o kúrios (Dánao). En la última llegada, el segundo regreso de Dánao, la compañía de los Argivos tiene dos efectos: arribando con escolta, el padre concentra la protección natural y la civil; la condición masculina y plural de los guardias representa, a su vez, un nuevo conflicto para las jóvenes.

A continuación, presentamos una descripción y breve análisis de cada uno de los cuatro movimientos:

Movimiento $1=$ súplica divina (1-175). La obra comienza con la llegada de las Danaides desde Egipto a Argos. Su aproximación al lugar sagrado, demarcado por el altar, coincide con el comienzo del acto de súplica, dirigida a los dioses en primera instancia. El movimiento en su totalidad coincide con la escena de la oda inicial.

Movimiento 2 = súplica humana (176-709). Todo el movimiento comprende la obtención del derecho de asilo en Argos, que constituye el motivo de súplica de las Danaides hacia su destinatario terrenal. El segmento se inicia con la primera intervención discursiva de Dánao, que imprime en la obra el ritmo agitado y el clima de ansiedad (176-233) ${ }^{42}$ y culmina al terminar la oda central (625-709), que encauza la desesperación inaugurada por el anuncio hacia el estado de júbilo y sosiego. Tras la escena de preparación, la concreción del objetivo involucra la representación de otras dos escenas. La primera (234-599) resulta ser solamente la escenificación de un conflicto entre las jóvenes y el rey, quien manifiesta su decisión de derivar la definición de su dilema (cólera de Zeus Suplicante us. guerra con los hijos de Egipto) a otro agente, el pueblo de Argos. Esta primera parte culmina con la segunda oda coral (524-599), en la que las Danaides exteriorizan su desespero ante la determinación de Pelasgo. La segunda escena (600-624) se inaugura con el sorpresivo primer regreso de Dánao (600), que anuncia la resolución del conflicto, y finaliza con la manifestación de felicidad inspirada por la noticia, constituida por la oda central (625-709).

Movimiento 3= amenaza efectiva (710-979). El movimiento entero abarca la puesta a prueba del beneficio de asilo recientemente obtenido. Tras haber sido persuadida la ciudad de recibir a los suplicantes, se inicia un nuevo núcleo temático, análogo al segundo movimiento ${ }^{43}$ : Dánao anuncia la llegada del enemigo (710-733) y se muestra la reacción de las jóvenes ante la noticia (734 y ss.). Después de la escena de preparación (710-835), la nueva contingencia también se desdobla en dos escenas. Pero, a diferencia del segundo movimiento, en el tercero se pone el acento en la angustia durante la espera de la llegada, que se dilata para justificar 
una nueva expresión cantada de las pasiones: la cuarta oda (776-824) ${ }^{44}$. En la primera escena, de manera idéntica que en la escena con el amigo, las Danaides enfrentan solas al heraldo de los Egipcios, que llega a las inmediaciones del eje del altar en el verso 82545. Tiempo después del grito desesperado por ayuda (835) promovido por la absoluta indefensión de las jóvenes, arriba súbitamente Pelasgo, que inicia la segunda escena (911-979). En ella, el conflicto iniciado por el segundo anuncio de Dánao se resuelve con la expulsión del heraldo (953) y la ratificación de Pelasgo y los ciudadanos argivos como protectores civiles de las Danaides, que ofrecen alternativas de alojamiento $(954-965)^{46}$. Sobre el final del movimiento, la novedad reside en la preparación para un nuevo regreso de Dánao (966-979) ${ }^{47}$.

Movimiento $4=$ nuevo conflicto (980-1073). El último movimiento se inaugura con el segundo regreso de Dánao (980) y finaliza cuando, dejando la orchéstra vacante, Dánao, Danaides y Argivos parten hacia la ciudad de Argos (1073). Este último segmento de la secuencia está integrado por el discurso de Dánao, que (re)ins-taura el tema de las responsabilidades femeninas (980-1017), y la performance de la oda final (1018-1073), que interacciona con el himeneo y coloca en el foco de atención el postergado tema del matrimonio. Durante la oda final dinámica (1062-1073), la acción de marcha cobra sentido en relación con la llegada inaugural (oda inicial dinámica, 1-39): las suplicantes han conseguido protección y parten junto a Dánao, de la misma manera como llegaron, pero en compañía de representantes de la ciudad. El cotejo entre el primer y cuarto movimiento es significativo desde el punto de vista visual: eísodoi opuestos (territorio peligroso us. región segura ${ }^{48}$ ) y un participante colectivo nuevo, que representa el éxito de la súplica que ha comenzado en la oda inicial, pero que, simultánea y paradójicamente, simboliza otro conflicto: la relación de las Danaides con el género masculino.

El diagrama que presentamos de la estructura de la obra evidencia que, además de la simetría generada por los anuncios extendidos, el equilibrio compositivo alcanza la totalidad de la propuesta dramática. En el verso 710, primer verso del segundo anuncio extendido que instaura la peripecia, la obra se divide en dos mitades espejadas, en las que se oponen el primer movimiento con el cuarto y el segundo con el tercero, estos últimos compuestos por una etapa de conflicto y una de resolución, e introducidos por una escena de preparación. En el diagrama, la representación de los movimientos de llegada al eje vertical señalado por el altar muestra que los acontecimientos peligrosos son los que provienen de Egipto, mientras que aquellos que ofrecen posibilidades de solución provienen de Argos. La presencia de los Argivos en el cuarto movimiento, que constituye la irregularidad en la correspondencia con el primer movimiento, es la que suscita el efecto extraordinario, corporizando el nuevo conflicto. Asimismo, según lo representa el esquema, las llegadas de los personajes determinan circunstancias alternadas de peligro y salvación, excepto el segundo regreso de Dánao que, junto a los Argivos, combina ambas alternativas, componiendo una instancia de salvación relativa. De este modo, el análisis de los movimientos de llegada de Suplicantes permite comprobar la aseveración de WILES: «Lateral oppositions are fundamental to interpretation of what the play means or meant, and it seems important to explore what symbolic charge the two sides had for the Greek spectator ${ }^{49}$. 


\section{Conclusión}

Hemos presentado un esquema compositivo de Suplicantes que permite aprehender la técnica estructural de esta obra en particular, sin atender a ningún molde preestablecido, normado y artificial. Nuestro enfoque de la arquitectura de la obra ha tenido en especial consideración el trabajo de TAPLIN sobre la puesta en escena trágica, que revolucionó los estudios performativos al demostrar la trascendencia de los movimientos escénicos de entrada y salida de los personajes. Sin embargo, dada la singularidad de Suplicantes en cuanto al protagonismo coral y el predominio lírico, hemos logrado afirmar que el análisis de su estructura impone revisar la propuesta de TAPLIN y considerar las odas corales como parte sustancial de los actos.

Sobre la base de estos presupuestos, propusimos para esta tragedia un esquema compuesto por cuatro movimientos, definidos por las "llegadas fundamentales" de los actores-personajes a las proximidades del altar, centro dramático de la escena, ocupado por el coro protagonista. El diagrama que hemos diseñado permite recrear y visualizar la simetría estructural de la obra generada por los anuncios extendidos, evidente dispositivo arquitectónico en función de su especularidad, y como recurso de preparación, ya que anticipa las escenas de metabolaí (peripecia y reconocimiento) y de agón ${ }^{50}$, es decir, las de mayor tensión dramática. Asimismo, el diagrama expone el carácter dramático y cardinal de las odas corales (fundamentalmente la inicial, central y final) y la trascendencia de la configuración espacial en relación con los personajes.

Suplicantes pone en evidencia no solo la trascendencia de la coralidad, sino también la singularidad de cada obra, abriendo un camino de análisis espacial para el resto de los dramas clásicos conservados.

\section{Bibliografía}

BAKKER, E. J. (2017) Mnemosyne Supplements. Authorship and Greek Song: Authority, Authenticity and Performance. Studies in Archaic and Classical Greek Song 3. Leiden: Boston.

BELlONI, L. (2006) Le Danaidi, Pelasgo, il nomos: note minime sulle «Supplici» di Eschilo. MD 57, 185-194.

BoweN, A. J. (2013) Aeschylus Suppliant Women. Edited with a translation, Introduction and commentary. Oxford: Editorial.

BowRA, C. M. (1933) Ancient Greek Literature. London: Editorial.

CALAME, C. (2017) La tragédie chorale: poésie grecque et rituel musical. Mondes anciens. Paris: Editorial.

Dupont-Roc, R. et Lallot, J. (1980) Aristote. La Poétique. Texte, traduction, notes, Paris.

FeRnández DeAGuSTINI, M. DEL P. (2016a) "Una aproximación a la sintaxis espacial en Suplicantes de Esquilo" Synthesis 23, pp. 49-70. 
(2016b) "Lo femenino en la literatura ateniense del periodo clásico. Suplicantes de Esquilo: la mujer ruiseñor" Phoînix, pp. 51-71.

(2017a) "El sentido de la forma. Los 'anuncios extendidos en Suplicantes de Esquilo", To $\varepsilon \lambda \lambda \eta v \iota \kappa o ́ ~ \beta \lambda \varepsilon ́ \mu \mu \alpha$ 2, UERJ, Río de Janeiro, Separata, 12, pp. 1-13 (separata 4)

- (2017b) "Identidad ritual y performance: un análisis de los primeros dos versos de Suplicantes de Esquilo" Calíope 34, pp. 4-22.

— (2017c) “Suplicantes de Esquilo. ¿Una trama primitiva?” Limes 28. pp. 39-61.

(2018a) "Drama, lírica e interacción genérica: la oda central de Suplicantes de Esquilo (625709)” Revista de Estudios Clásicos 45, pp. 101-146.

(2018b) "Suplicantes de Esquilo. Ritual de mujeres migrantes: la oda inicial como performance de la alteridad", en De Sousa Lessa, F. (comp.), Literatura y sociedad en la Grecia antigua (pp. 65-84) Río de Janeiro: UFRJ.

_ (2019a) "Ser mujeres, estar subordinadas. El mapa de coordenadas masculinas en Suplicantes de Esquilo", en Zecchin, G. (comp.), Literatura y sociedad en la Grecia antigua. La Plata: UNLP. (En prensa).

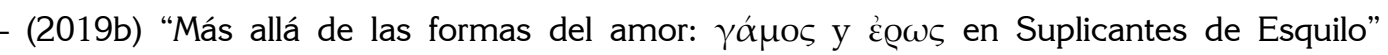
Euphrosyne (en prensa).

Garvie, A. (1978) "Aeschylus' Simple Plots”, en Dawe, R. D., Diggle, J., Easterling, P. E. (Eds.), Dionysiaca. Nine Studies in Greek Poetry by Former Pupils Presented to Sir Denis Page on His Seventieth Birthday (pp. 63-86). Cambridge: Editorial.

(2006) Aeschylus' Supplices. Play and Trilogy (corrected ed.), UK.

GoldHILL, S. (1990) The Great Dionysia and Civic Ideology. En Winkler, J. J.; Zeitlin, F. Nothing to do with Dionysos? Athenian Drama in its Social Context, (pp. x-x). Princeton: Editorial.

Johansen, F. H. -WhiTtLe, E. (eds.) (1980) Aeschylus. The Suppliants. Copenhagen: Editorial.

KITTO, H. D. F. (1939) Greek Tragedy. A Literary Study. London: Editorial.

(1956) Form and Meaning in Drama. London: Methuen.

(2011) Greek Tragedy: a Literary Study (revised); with a foreword by Edith Hall. Oxon (reproducción de la tercera edición de 1961)

LATHAM, A. (2008) Diccionario enciclopédico de la música. México: FCE.

LEY, G. (2007) The Theatricality of Greek Tragedy. Playing Space and Chorus, Chicago: Editorial.

Lloyd-JONES, H. (1964) "The Supplices of Aeschylus: The New Date and Old Problems", Ant.Class. 33, pp. 356-374.

Lobel, E.;Wegener, E. P.; Roberts, C. H. (1952) The Oxyrhynchus Papyri. Part XX (Nos. 2245-2287). London: Editorial.

MURRAY, G. (1955) Esquilo: el creador de la tragedia. Madrid: Editorial.

ReHM, R. (2002) The Play of Space: Spatial Transformation in Greek Tragedy. Princeton: Editorial.

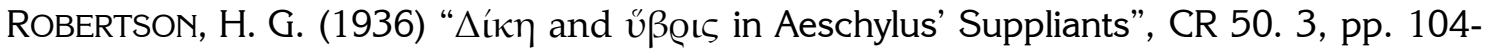
109. 
SANDIN, P. (2002) "Critical notes on Aeschylus", Eranos 100.2, pp. 146-160.

SHEPPARD, J. T. (1911) "The First Scene of the Suppliants of Aeschylus”, CQ 5.4, pp. 220-229.

Sommerstein, A, H. (2008) Aeschylus. Persians, Seven against Thebes, Suppliants, Prometheus Bound. Cambridge-Massachusetts-London: Editorial.

SwIFT, L. (2010) The Hidden Chorus: Echoes of Genre in Tragic Lyric. Oxford-New York: Editorial.

TAPLIN, O. (1977) The Stagecraft of Aeschylus. The Dramatic Use of Exits and Entrances in Greek Tragedy. Oxford: Editorial. (1978, 2003) Greek Tragedy in Action. London-New York: Editorial.

TucKER, T. G. (1889; 2007) The Supplices of Aeschylus. Montana: Kessinger Publishing. UBERSFELD, A. (1981) L'école du spectateur.Paris: Editorial.

WILES, D. (1997) Tragedy in Athens. Performance Space and Theatrical Meaning. Cambridge: Editorial.

WiLsOn, P. (2005) "Music”, en Gregory, J. (Ed.), A Companion to Greek Tragedy (pp. x-x). London: Editorial.

WOLF, E. A. (1958) “The date of Aeschylus' Danaid tetralogy”, Eranos 56.3-4, pp. 119-139.

YORKE, E. C. (1954) “The date of the Supplices of Aeschylus”, CR 4.1, pp. 10-11.

Notas

${ }^{1}$ El revolucionario fragmento conservado en P. Oxy. 2256.3, adjudicado a Aristófanes de Bizancio, fue publicado por primera vez por LOBEL-WEGENER-ROBERTS (1952:30). Para una discusión completa de este fragmento, GARVIE (2006:1-28). Dos artículos específicamente dedicados a la polémica sobre la datación de Suplicantes son los de YORKE (1954) y WOLF (1958).

2 TUCKER (1889: xvi). Entre quienes han considerado la pieza como una extravagancia primitiva, ninguno dudó en aceptar esta multitud de extras. La posición sobre la puesta en escena estuvo determinada por la manera en que ha sido concebida la relación entre palabras y acción en el teatro griego clásico. Los estudios de fines del siglo XIX y principios del XX tendieron a asumir que la performance aspiraba a ser realista; en el extremo opuesto, un grupo de estudiosos, a mediados de siglo XX, propuso equiparar el drama griego con las convenciones no naturalistas de los dramas japoneses Noh y Kabuki, cuestionando si los más simples movimientos u objetos escénicos habrían sido representados miméticamente. Entre el teatro realista y el de discursos, TAPLIN (1977: 31) ha iniciado un camino más equilibrado, poniendo su atención sobre las "acciones significativas" del drama, cuya trascendencia se infiere del texto. Para más información acerca de las conjeturas sobre la espectacularidad en Suplicantes, TAPLIN (1977: 202 y ss.).

3 Otros críticos de reconocida tradición en el estudio de Suplicantes son ROBERTSON (1936:104), que opina que su acción y caracterización son simples y rudimentarias; BowRA (1933:81) que simplifica el argumento: «Such action as there is consists of their [the suppliants'] efforts to secure protection, and the arrival of a herald from Egypt announcing 
the presence of the rejected suitors». MURRAY (1955) sintetiza el argumento como un pedido de protección dirigido a Pelasgo, quien refiere la pregunta al dêmos. En los dos últimos casos, la sinopsis revela que, para estos estudiosos, el drama tenía lugar a partir de la llegada de Pelasgo, lo cual deja fuera no solo la coyuntura que hace de la obra una tragedia, sino también al coro como personaje protagónico. Otras numerosas descalificaciones de la calidad dramática de Suplicantes están compiladas en el artículo de SHEPPARD (1911:222223). No obstante, el autor resultó ser una notable excepción entre los estudiosos de su época por intentar rescatar la obra de este destino crítico a partir de la puesta en valor de la que él considera la "primera escena" de la tragedia: la oda inicial.

${ }^{4}$ Esta premisa domina otro reconocido trabajo de KITTO, Form and Meaning in Drama, de 1956.

${ }^{5}$ KITTO (1961:3; reedición de 2011). Más tarde surgieron otras miradas sobre la obra. GARVIE (1978 passim), también consciente de la complejidad de Suplicantes, estudió el rol de la anticipación, el suspenso y la sorpresa en las tragedias de Esquilo en general. Entre los autores actuales, Belloni (2006:186) rescata la excelencia de la tragedia: «...la drammaturgia di questa tragedia 'semplice'; almeno secondo i princìi che noi ci proviamo a desumere dalla Poetica di Aristotele per una $\dot{\alpha} \pi \lambda \tilde{\eta} \pi \rho \tilde{\alpha} \xi \iota \varsigma$ sprovvista di una vera e propria peripezia, e dotata di uno spessore corale che sembra volto a integrare, in forme diverse, le carenze dinamiche dellintreccio. Ma se queste ultime, a un'analisi più attenta, non risultano tali nelle Supplici -soprattutto nello spazio compreso fra la preghiera celebrativa di Argo e il sopraggiungere dell'araldo egizio (vv. 625-709, 835 ss.), dove le sequenze sceniche potrebbero anche rivelare una tecnica più raffinata».

${ }^{6}$ WILES (1997: 1-22, especialmente pp. 9-10).

${ }^{7}$ WILES (1997: 87): «Plato, unlike Aristotle, grew up in Athens and experienced fifth-century drama. He conceives tragedy as an event rather than a text». El autor amplía este punto de vista tanto en la introducción de su libro (pp. 6-9) como a lo largo del capítulo cuarto.

${ }^{8}$ UBERSFELD (1981: 64). Sobre la dicotomía plataforma/proscenio, WILES (1997: 15).

${ }^{9}$ Todos los argumentos, estilísticos e históricos, vinculados con la fecha temprana de Suplicantes son expuestos con detalle y refutados por GARVIE (2006: 29-162). Asimismo, pueden consultarse JOHANSEN-WHITTLE (1980: I.25-29) y LLOYD-JONES (1964).

10 TAPLIN (1978: 14): «Even this oversimplified pattern would be far from monotonous, thanks to the wide variety of kinds of delivery permitted within the acts. Also the acts may vary greatly in length (we have, in fact, acts ranging from about 20 to 500 lines), and so might the choral songs between the acts (from some 20 lines up to 200 or so); also there may be exits and entrances during the course of the acts, though very seldom in rapid sequence».

${ }^{11}$ TAPLIN (1978: 14). El subrayado es nuestro.

${ }^{12}$ Si bien Taplin delimita un "acto" según la existencia de un movimiento de ingreso previo y uno de egreso posterior, en su propuesta la definición de "acto" es muy similar a la de "episodio" de Poética 1452b 20-21: "una parte completa de tragedia en medio de canciones corales enteras». El subrayado es nuestro.

${ }^{13}$ Cfr. FernándeZ Deagustini (2016b, 2017b, 2018a y b).

${ }^{14}$ WILES (1997: 87-132) dedica dos capítulos a demostrar la trascendencia de la coralidad en la tragedia, The mimetic action of the chorus y The chorus: its transformation of space. 
${ }^{15}$ La comprensión del drama clásico ateniense ha sido revigorizada por la exploración de los aspectos no verbales de la comunicación dramática, como el espacio, el sonido, la música y la danza (TAPLIN 1977; WILES 1997; REHM 2002 para el espacio; LEY 2007 para la danza; WILSON 2005 para la música). La inclusión de los certámenes teatrales en los cuales se desarrollaban las celebraciones rituales en honor a Dioniso señaló secuencias específicas de eventos anteriores y posteriores a la representación de las obras (GOLDHILL 1990). Sin duda, este giro ha sido representado por la publicación del ya clásico volumen Nothing to do with Dionysos?, significativamente subtitulado Athenian Drama and its Social Context (WINKLER- ZEITLIN 1990). Recientemente, se destacan las publicaciones de SWIFT (2010), CALAME (2017) y BAKKER (2017), entre otras.

${ }^{16}$ WILES (1997:125).

${ }^{17}$ El diálogo en Suplicantes se da predominantemente entre el coro protagonista y algún actor, a excepción de dos intercambios entre actores: Pelasgo-Dánao (490-503) y Pelasgoheraldo (911-953). En relación con el discurso lírico, el canto es unívoco en las cinco odas, pero no siempre solitario, ya que en las odas inicial, central y final esa expresión se da en presencia de Dánao.

${ }^{18}$ Tomamos este concepto del de WILES, vertical axis (1997: 175-186), al que dedica un capítulo completo. Wiles indica que el teatro griego clásico disponía de pocos recursos para mapear la dimensión vertical, pero que este eje era crucial para demarcar el universo tripartito de los inmortales, los mortales y los muertos.

${ }^{19}$ Cfr. FeRnáNDEZ DeAgustini (2016a).

${ }^{20}$ TAPLIN (1977: 53).

${ }^{21}$ Nos remitimos al subtítulo de su libro, Performance Space and Theatrical Meaning.

${ }^{22}$ Es fundamental la relación que WILES (1997: 124), oponiéndose a Taplin, propone entre las odas corales y los desplazamientos de ingreso y salida de los personajes: «In a theatre as large as the Theatre of Dionysus, with long slow entrances through the eisodoi, we should not conceive of the chorus finishing its dance in order for a character subsequently to make an entry. The last lines of choral odes overlapped entries, and the first lines overlapped exits».

${ }^{23}$ Acorde con la premisa fundamental de que las instrucciones escénicas significativas están implícitas en las palabras, TAPLIN (1977: 28) determina: «The entry of a character (as opposed to an actor) occurs, in effect, when the character newly engages in or impinges on the words and action of the play-and his exit when he withdraws or disengages from them» (p. 8). Por lo tanto, se considera que un personaje "llega" cuando habla o cuando se le dirige la palabra por primera vez. La partida de los personajes se determina después de las últimas líneas pronunciadas por el personaje en cuestión. En cuanto a los personajes "menores", como es el caso de los Argivos en Suplicantes, si su entrada no es explícitamente subrayada por quienes se encuentran en escena, debe suponerse que han entrado junto a un personaje superior, al cual se encuentran unidos (Pelasgo o Dánao).

24 TAPLiN (1977: 7) propone esta distinción. LeY (2007: 3) critica la influencia de la terminología de Taplin en la última generación de estudiosos, por estimular el uso de "entrada" y "salida" para un espacio teatral para el cual estos conceptos resultan inapropiados: "Taplin also wrote in passing of "arrivals and departures", and the terminology I advocate is fourfold, taking account of those who "arrive" in the playing space or "leave" it 
along the side approaches (known diversely as parodoí or eisodoí), and those who either "appear" from or "enter" the skené».

${ }^{25}$ TAPLIN (1977:192): «There are, however, five surviving plays -A. Hik, Eum; S. OC; E. Hkld, Hik- which are largely constructed around the reception of fugitive suppliants by a virtuous city or ruler and the repulse of their aggressive pursuers. Though this recurrent plot is given many variations it is sufficiently fixed in its main elements to be regarded as a kind of 'story pattern'. The story pattern tends to bring with it recurrent roles and situations, and hence also recurrent types of exit and entry: the entry of the fugitives, of the protector, of the pursuer, the re-entry of the protector to the rescue, and the final departure to safety».

${ }^{26}$ Cfr. FERNÁNDEZ DEAGUSTINI (2017a). La expresión extended announcements pertenece a TAPLIN (1977).

${ }^{27}$ TAPLIN (1977: 10): «But to a large degree the significance of an entry or exit depends on what has passed before it, on the preparation for it. It is the preparation which creates expectation and which puts the event into its dramatic context, and hence gives it work to do in the play».

${ }^{28}$ Según TAPLIN (1977: 12), las técnicas de sorpresa eran excepcionales en la tragedia griega, pues la expectativa resulta una fuerza emocional más poderosa que esta.

${ }^{29}$ Las ediciones de la obra utilizadas para determinar los versos de cada una de las llegadas son las de SOMMERSTEIN (2008) y BOWEN (2013). Sobre la dificultad que proponen algunos de estos pasajes, cfr. FERNÁNDEZ DEAGUSTINI (2016a).

${ }^{30}$ El orden cronológico de estos arribos no es fijo, sino que varía según la propuesta dramática en cada caso.

${ }^{31}$ Cfr. FeRNÁNDEZ DEAguSTINI (2017b y 2018b).

32 El poder teatral de estos ingresos prolongados a través de los eísodoi ha sido ampliamente demostrado por TAPLIN (1977: 451). Taplin ha reconocido que el dramaturgo, en cada obra dada, establece dos direcciones precisas y diferentes para los eísodoi y que esta disposición habría sido útil en una obra como Suplicantes de Esquilo, donde «el conflicto total de la obra» habría adquirido una forma concreta en ambas direcciones. Como señala WILES (1997: 134), "The issue is left open and unexplored in The Stagecraft of Aeschylus, though a later article on Sophocles proposes that in suppliant plays generally one eisodos leads to the safety of a receiving city and the other leads to danger». A pesar de esta observación, Wiles tampoco se ocupa de analizar esta tragedia.

${ }^{33}$ Cfr. FeRnándeZ DeAgustini (2019a:76- 80).

${ }^{34}$ Sobre otras características irregulares del ingreso de Dánao a escena, TAPLIN (1977: 224229). El autor dedica varias páginas a exponer su preocupación sobre la transición entre la salida de Pelasgo y el ingreso de Dánao (974-980). Por un lado, es acertada su observación acerca del apurado manejo de este cambio de escena en términos de viabilidad técnica y de manipulación de la temporalidad en escena. Efectivamente, parece inverosímil el tiempo con el que cuenta el rey para buscar al padre de las Danaides, tal como ellas lo solicitan en el verso 967, y parece escaso el tiempo que el actor tiene para cambiar el rol del heraldo por el de Dánao (951-980). Por otro lado, Taplin deja sin resolver una irregularidad en la composición que cataloga como "malformación" en el cuerpo de la pieza (p. 229): se trata de una escasa cantidad de versos en la cual el escenario permanece vacante de actores y sin canción estrófica del coro. Dice TAPLIN (1977: 227): «It is very rare in Greek tragedy to 
have a stage vacant of actors except during act-dividing songs. I can think of no other act division in Aeschylus or all Greek tragedy whose technique resembles Hik. 974-80 (or 974/5). There is no reason why the technique should not be unparalleled: but we must ask why it is unique, what is gained by it. There is no close dramatic connection between the two acts, no particular continuity: on the contrary, there is asyndeton, plain juxtaposition». Varios puntos fallan en la apreciación de Taplin para concluir que se trata de una «apresurada, torpe y malhecha transición» (p. 229) impropia de la tragedia griega, donde los movimientos son pocos y bien separados. En primer lugar, su aproximación a la tragedia mantiene el principio de la alternancia entre discurso de actores y canto coral. En segundo lugar, por concentrarse en el coro de servidoras, pierde de vista la importancia dramática que cobra el segundo regreso de Dánao junto a los guardias, dado que, por su fuerte presencia escénica, los Argivos generan un acentuado corte entre escenas pero, simultáneamente, funcionan como un nexo de continuidad, tanto por su anterior participación visual como por la casi inmediata intervención discursiva con que se los destaca pocos versos más tarde. Los tópicos matrimonio-súplica-matrimonio están encadenados. El asíndeton cumple con su función de subrayar y significar la trascendencia del cambio de tópico. Finalmente, no resulta un detalle menor en la composición de este cambio de escenas el hecho de que Pelasgo se retire silenciosamente, mientras que Dánao llegue acompañado por los guardias, como si fuera un rey. Sin duda, una canción en medio de ambos acontecimientos hubiera hecho perder de vista ese significativo contraste otorgado a ambos personajes.

${ }^{35}$ Optamos por dejar aquí el concepto original griego, por los problemas de traducción que supone en el contexto de Suplicantes.

${ }^{36}$ Para el análisis particular del tema del matrimonio, los debates relacionados en torno a esta obra y el planteo de una posibilidad de interpretación diferente, cfr. FERNÁNDEZ DEAGUSTINI (2019b).

${ }^{37}$ TAPLIN (1977: 219) señala, a propósito de esta llegada de Pelasgo: «In drama the boé makes for an exciting rise in tension (and in the volume of the sound), and it provides a convincing and integral entry motivation». Sin embargo, a pesar de que Taplin no lo señale, la motivación no impide la posibilidad de la sorpresa, ya que Pelasgo llega ochenta versos más tarde e interviene impetuosamente, probablemente agregando estrés a la escena con su paso apresurado.

${ }^{38}$ Cfr. FERNÁNDEZ DEAgUSTINI (2019b).

${ }^{39}$ WILES (1997:134).

40 TAPLIN (1977:209) diferencia ambas escenas considerando exclusivamente la manipulación de los movimientos de ingreso y salida: «At the equivalent point in Hik, on the other hand, there is no entry at all: instead Danaus describes for a second time what he sees in the distance. So not only is there no exit before the song 625-709, there is no entry after it». Sin embargo, es necesario recordar que, tras los dos anuncios, también el tiempo dramático se emplea de manera diferente. La llegada de Pelasgo se produce inmediatamente después de que las Danaides han tomado la posición ritual indicada por Dánao. Su padre, además, permanece junto a ellas. La llegada del enemigo, en cambio, se demora. Dánao anuncia, además de la mala noticia, que debe alejarse en busca de ayuda. Solas en la orchéstra, las Danaides exteriorizan su temor cantando la cuarta oda. En medio del clima generado por un canto que interacciona con el treno se concreta la llegada del heraldo. 
${ }^{41}$ LATHAM (2008: 986).

${ }^{42}$ La apreciación de la participación escénica de Dánao es distinta a la de TAPLIN (1977: 198), quien sostiene que la intervención de Dánao debe ser considerada como virtualmente equivalente a una entrada. Los argumentos del autor son dos: "The structural break at this point is marked not only by the change from song to speech but also by a new turn of events which could normally only be achieved by an entry (also 710)». Desde nuestro punto de vista, el cambio de discurso cantado a discurso hablado modifica el ritmo de la obra, pero no significa un quiebre. La continuidad está dada por el aspecto visual, ya que, desde que llega, Dánao se encuentra en la misma posición espacial, en la estructura elevada del altar, mirando hacia el horizonte. Por lo tanto, el espectador, desde el comienzo de la oda, está esperando novedades por parte de Dánao. El anuncio tampoco implica una modificación radical en el accionar de las Danaides, dado que sus preocupaciones se dividen entre los dioses y los hombres que se aproximan. El centro de atención virará completamente, de los dioses al hombre, una vez que Pelasgo se haga presente en el verso 234. Las escenas de preparación, por lo tanto, articulan dos movimientos.

${ }^{43}$ Además de las semejanzas ya señaladas, por segunda vez no hay ingreso de ningún personaje después de la canción (1-175; 625-709).

${ }^{44}$ TAPLIN (1977: 60) insiste en que, cada vez que el patrón estructural se altera, es necesario intentar descubrir el propósito de la variación. No obstante, esta correcta apreciación teórica, el autor no advierte que, más allá de las coincidencias entre ambas "escenasespejo", en la segunda la llegada se demora de manera extraordinaria después del anuncio. La deliberada intención del dramaturgo de generar, junto a la expectativa, pánico y angustia entre los destinatarios internos y externos de la noticia, pasa inadvertida para el estudioso.

${ }^{45}$ Las escenas con el amigo y el enemigo coinciden desde el punto de vista discursivo, pero no desde el visual. La desprotección de las Danaides se acentúa en el tercer movimiento, en el cual las Danaides no solo enfrentan al heraldo, sino que lo hacen sin contar con la presencia física de su padre. En el segundo movimiento, en cambio, Dánao contempla la súplica desde el altar y, luego, interviene para solicitar protección armada a Pelasgo.

${ }^{46}$ Sobre la edición y sentido de los versos 957-961, que concierne las opciones que Pelasgo propone a las Danaides, SANDIN (2002: 150-152).

${ }^{47}$ A diferencia de las dos instancias previas de preparación, que fundan acontecimientos completamente nuevos (176 y ss.; 710 y ss.), la gestación de la tercera llegada está justificada por la invitación de Pelasgo para que las Danaides vayan a la ciudad. El pedido de las Danaides por Dánao, entonces, se asocia con el tema de la súplica y la seguridad lograda, propios del tercer movimiento. El hecho de que la llegada de Dánao inaugure un nuevo conflicto es desconocido para el espectador hasta el momento en que el padre profiere su último discurso. Por este motivo, el cuarto movimiento no comienza con la preparación de la llegada, sino cuando esta se concreta, en el verso 980.

${ }^{48} \mathrm{El}$ contraste y sentido de las direcciones offstage se instala en los anapestos iniciales cuando las Danaides cuentan la huida de su tierra y el arribo a Argos (1-22), suplicando ser recibidas y que los Egipcios sean rechazados. Lógicamente, este juego de opuestos es aprovechado en la composición de los anuncios extendidos.

${ }^{49}$ WILES (1997: 142).

${ }^{50}$ Cfr. FeRnáNDEZ DeAgustini (2017c). 